Communications in Physics, Vol. 30, No. 2 (2020), pp. 111-122

DOI:10.15625/0868-3166/30/2/13934

\title{
SIZE AND LAYER DEPENDENCE OF HYBRID GRAPHENE/H-BN MODELS UPON HEATING
}

\author{
NGUYEN THI THUY HANG ${ }^{1,2, \dagger}$ \\ ${ }^{1}$ Laboratory of Computational Physics, \\ Faculty of Applied Science, Ho Chi Minh City University of Technology, Ho Chi Minh City, \\ 268 Ly Thuong Kiet Street, District 10, Ho Chi Minh City, Vietnam \\ ${ }^{2}$ Vietnam National University Ho Chi Minh City, \\ Linh Trung Ward, Thu Duc District, Ho Chi Minh City, Vietnam \\ ${ }^{\dagger}$ E-mail: hangbk@hcmut.edu.vn
}

Received 12 July 2019

Accepted for publication 17 March 2020

Published 15 May 2020

\begin{abstract}
Different models contained graphene layer are studied via molecular dynamics simulation. Models are heated up from $50 \mathrm{~K}$ to $8000 \mathrm{~K}$ via Tersoff and Lennard-Jones potentials to have an entire picture about the evolution of graphene layer in the models upon heating. Various thermodynamic quantities, structural characteristics, and the occurrence of liquidlike atoms are studied, such as, the total energy per atom, the heat capacity per atom, the radial distribution functions, and the appearance of liquid atoms upon heating. The phase transition exhibits the first order. The melting point of graphene layer depends on the number of layers in the models while it does not depend on the size in the range of this study. The melting process of hybrid graphene and hexagonal boron nitride ( $h-B N)$ satisfies the first step towards Devil's staircase type phase transition. The melting point of hybrid graphene/h-BN is close to the one of experiment of graphite.
\end{abstract}

Keywords: Hybrid graphene/hexagonal Boron Nitride models; Layer dependence; Melting range; Liquidlike atoms; Layer dependence;.

Classification numbers: 81.07.Nb. 


\section{INTRODUCTION}

As well known hexagonal Boron Nitride (h-BN) and graphene have many similar physical properties and analogous structure such as strong mechanical properties, high chemical, thermal stability, and lattice constant $[1,2]$. Moreover, due to the similar lattice parameters of graphene and h-BN, h-BN would be a suitable substrate to synthesize graphene [3-5]. Therefore, the hybridization of h-BN with graphene is studied intensively to find new physical characters such as semiconducting properties as well as to create new materials.

Recently, investigation in semiconducting graphene using $\mathrm{B}$ and $\mathrm{N}$ atoms has been intensively studied [6-8]. In addition, graphene and h-BN is assembled into heterostructures to study the band gap, various semiconducting structures, and mechanical properties [9-15]. Moreover, hybrid graphene/h-BN structure is considered as one of the most feasible methods to control the semiconducting properties of graphene. In addition, h-BN is an insulator which can be an ideal dielectric to build metal|h-BN|graphene field-effect devices [16]. Related to synthesize graphene, $\mathrm{h}-\mathrm{BN}$ is a perfect substrate to grow graphene using chemical vapour deposition method [17]. However, for the catalyst-free approach, the results are poor quantity due to the difficulties in control over the shape and crystallinity of the graphene [18-22]. Therefore, to achieve a high-quality graphene/h-BN different foils are used such as copper, platinum [23-26]. As for new hybrid nanomaterials, the combination of $\mathrm{B}, \mathrm{N}$, and $\mathrm{C}$ can make new interesting physical properties such as: i) the $\mathrm{B}-\mathrm{N}$ and $\mathrm{C}-\mathrm{C}$ bonds have tendency to separate in the whole $\mathrm{BCN}$ systems [27]; ii) The combination of graphene and h-BN can create a new structure consisting of hybridized phases of h-BN and graphene (h-BNC) [28]

Based on the discussion above, new materials with suitable physical properties can be produced. In order to make out of hybrid materials or synthesized materials, the melting points as well as the information close to and exceeding the melting point play an important role. Based on those information, the conditions to synthesize materials can be established, such as, thermal gradient, melting zone, and homogenization temperatures. However, as for graphene, it is difficult to study the melting process experimentally because of high stability of the graphene structure. Therefore, molecular dynamics simulation is a suitable choice to study the structural evolution of graphene upon heating.

In this study, the influence of temperature on various models contained graphene layer is studied to have an entire picture about the evolution of graphene layer of hybrid models upon heating. The thermodynamic properties are studied via molecular dynamics simulation. The structural characteristics are studied based on the occurrence of liquidlike atoms. The atomic mechanism is studied based on the occurrence/growth of liquidlike atoms, the formation of clusters. Details about the calculations are showed in Sec. II. Results and discussions can be found in Sec. III. Conclusions are given in the last section of the paper.

\section{CAlculations}

There exist different empirical potentials to study several different bonding states of an atom as well as the chemical reactions such as Stillinger-Weber (SW) type potentials [29-32], embedded atom models (EAMs) [33,34], higher bond order potentials derived from tight binding models (TBBOPs) [35,36] reactive bond order (REBO) potential [37], and reactive force field [3840]. In this study, the model is a combination of two layers (graphene layer and h-BN layer) 
leading to using different potential: Tersoff potential is used to study graphene and h-BN layers while Lennard-Jones potential is applied for the interaction between the layers due to the weak interaction.

The Tersoff potential is used with various set of parameters for boron (B)-nitrogen $(\mathrm{N})$, and graphene [41-43], given below:

$$
E_{b}=\frac{1}{2} \sum_{i \neq j} f_{c}\left(r_{i j}\right)\left[a_{i j} f_{R}\left(r_{i j}\right)+b_{i j} f_{A}\left(r_{i j}\right)\right]
$$

in which, $r_{i j}$ denotes the distance from atom $i$ to atom $j$ and the sum runs over all atomic sites. The repulsive $f_{R}\left(r_{i j}\right)$ and the attractive $f_{A}\left(r_{i j}\right)$ terms are chosen similar to a Morse potential as proposed by Brenner [44]. The $f_{c}\left(r_{i j}\right)$ term represents a cutoff function, which calculates the number of neighbors and makes the potential to zero outside the interaction shell.

Because of weak interlayer interaction of graphene and h-BN sheets, Lennard-Jones potential [45] is used to describe van der Waals and Pauli repulsive potential, given below:

$$
E_{b}=4 \varepsilon\left[\left(\frac{\sigma}{r}\right)^{12}-\left(\frac{\sigma}{r}\right)^{6}\right]
$$

in which, $r$ is the distance between two atoms, while $\varepsilon$ is a parameter determining the depth of the potential well, $\sigma$ is the diameter of the atom (a length scale parameter of the atom determining the position of the potential minimum). The repulsive forces are described by the first term $\left(r^{-12}\right)$ while the attractive forces are studied by the second term $\left(r^{-6}\right)$. Parameters in LJ potential are determined by the method of Kang and Hwang [46].

In order to perform the calculations we use the software package Large-Scale Atomic/ Molecular Massively Parallel Simulator (LAMMPS), designed to solve various problems by the methods of classical molecular dynamics [47].

The simulation scenario starts with the initial crystalline hybrid graphene/h-BN models. Models with different number of atoms and layers are chosen such as two layers (graphene/h-BN) and three layers (graphene/h-BN/graphene) to have an entire picture about the evolution of hybrid graphene/h-BN models. These models have been relaxed in the isothermal-isobaric ensemble (NPT) for $5 \times 10^{5}$ molecular dynamics (MD) steps at $T_{0}=50 \mathrm{~K}$. In order to model two dimensional materials, periodic boundary conditions are applied along $\mathrm{x}$ and $\mathrm{y}$ axes while non-periodic boundary condition is along z-axis (in Cartesian Coordinates). Note that $1 \mathrm{MD}$ step takes 0.0001 picoseconds. Next, a temperature $T_{i}\left(T_{i}=8000 \mathrm{~K}\right)$ is chosen. This chosen temperature is higher than the melting point of free standing graphene [48] or h-BN [49] to observe the evolution of the models upon heating. The system is heated from the temperature $T_{0}$ to $T_{i}$ in the canonical ensemble. The heating rate is $10^{12} \mathrm{~K} / \mathrm{s}$.

We use Visual Molecular Dynamics (VMD) software for two dimensional (2D) visualization of atomic configurations [50]. The increase of temperature obeys the following expression: $T=T_{0}+\gamma t$. Here, $T_{0}=50 \mathrm{~K}$ and $\gamma$ is a heating rate, $t$ is a time required for heating. Models, obtained at each temperature have been relaxed at a given temperature for $6 \times 10^{5} \mathrm{MD}$ steps before analyzing the structural characteristics or $2 \mathrm{D}$ visualization. 


\section{RESULTS AND DISCUSSION}

To start with, layer dependence of melting process is analyzed to study the evolution of graphene upon heating. Two-layer (graphene/h-BN) and three-layer (graphene/h-BN/graphene) models with 5000 atoms/layer are presented. Total energy per atom is analyzed to study the phase transition of graphene layer (solid circle symbol - two layers and square symbol - three layers in Fig. 1). In general, the total energy per atom changes following three stages upon heating. Firstly, total energy increases linearly since the studying models remain in solid state (in the range of temperature below the melting point). Secondly, the total energy per atom has a sharp increase around the melting point of the model because the contribution of the anharmonic motion of atoms is strong enough leading to a massive collapse of a crystalline matrix. The total energy starts to deviate from the linear law in the melting range of temperature. This sharp increase of total energy per atom indicates the first order-like phase transition from solid to liquid states. Finally, the total energy per atom increases linearly again indicating that the model is in a liquid state. One can conclude that the type of phase transition does not depend on the number of layers in this range of study.

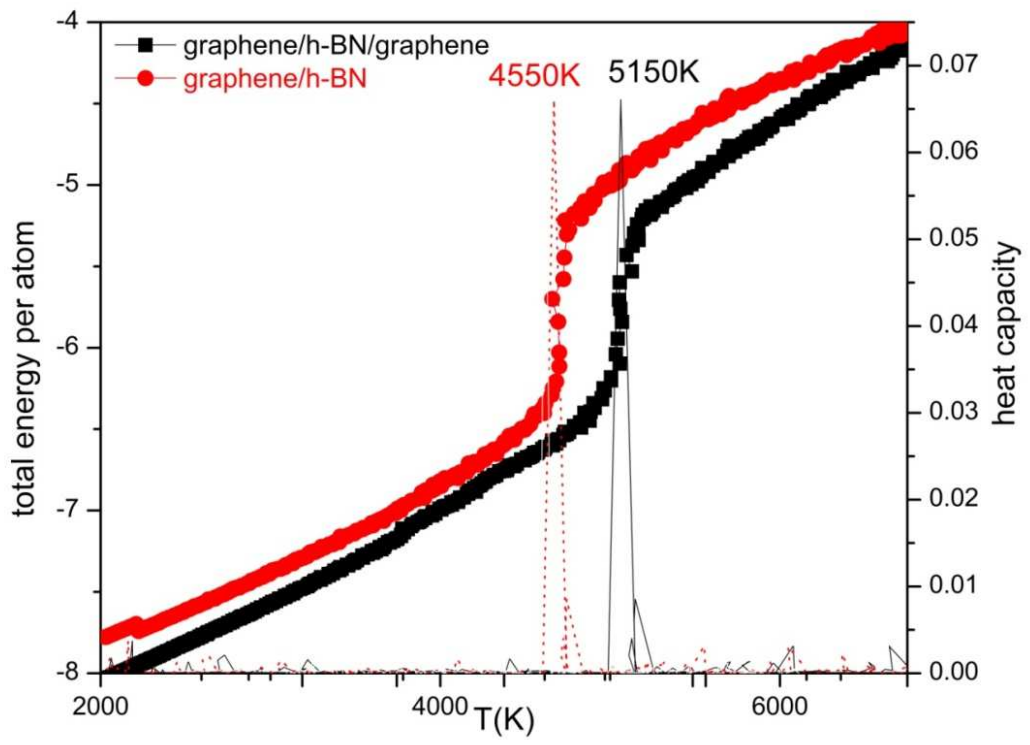

Fig. 1. Temperature dependence of total energy and heat capacity per atom of graphene layer: graphene/h-BN model (total energy - solid circle symbol, heat capacity - solid line); graphene/h-BN/graphene model (total energy - square symbol, heat capacity - dot line).

In term of temperature dependence of layer number, heat capacity at a constant zero pressure is approximately calculated via the simple relation: $C_{p}=\Delta E / \Delta T$. The behavior of heat capacity per atom of graphene layer is calculated to define the melting temperature (graphene/h-BN model - dot line, graphene/h-BN/graphene model - solid line in Fig. 1). As well known the peak 
of the heat capacity represents a sudden change in total energy which shows the first order-like phase transition from solid to liquid states in studied models. More details can be seen in Fig. 1 that the peak of graphene layer in graphene/h-BN model is $4550 \mathrm{~K}$ (dot line in Fig. 1) while the one of graphene layer in graphene/h-BN/graphene model is around $5150 \mathrm{~K}$ (solid line in Fig. 1). One can see that the peak of the heat capacity of graphene layer in graphene/h-BN/graphene model has tendency to shift to the higher range of temperature in comparison with the one of heat capacity of graphene layer in graphene/h-BN model. In conclusion, in the range of this study, the melting point depends on the number of layers in the model. Moreover, the increasing number of layers in model leads to decrease statistical noise.

The three dimensional (3D) view of the two-layer model with 5000 atom/layer is presented in Fig. 2 to have a visualization of the state of model at 4600K. It can be seen in Fig. 2 that the solid structure of graphene layer is almost broken (Fig. 2a) while the one of h-BN is still conserved (Fig. 2c). That leads to the conclusion that the melting process of hybrid material satisfies the first step towards Devil's staircase type phase transition [51].

It can be seen a significant difference in melting points between free-standing graphene which was studied in reference [48] (around 8000K) and graphene layer in hybrid models in this study (around $4550 \mathrm{~K}$ - graphene/h-BN and $5150 \mathrm{~K}$ - graphene/h-BN/graphene). To clarify this difference, some points related to free-standing graphene as well as hybrid materials are presented here.

Regarding free-standing graphene, there are some reasons leading to a high melting point in this case. First of all, the essential interactions between carbon atoms are very strong. Secondly, because of free-standing graphene, there are no any external interactions such as the van der Waals from other layers, which can affect on the hardness of graphene structures. Thirdly, the conditions of the simulation box in the reference [?]lso contribute an amount of temperature in the melting point of graphene.

As for hybrid materials, there are two types of interactions between two materials: van der Waals and chemical bonds. The former represents a weak interaction between two layers of materials which is usually described by Lennard-Jones potential [45] in simulation. Some types of materials such as graphite, Lennard-Jones potential is used to describe the weak interactions between graphene layers to make out of graphite. However, the interactions between atoms in a layer are very strong (chemical bonds), which lead to a high energy for breaking bonds between atoms in a layer in comparison to a low one for separating layers in graphite. The latter represents a strong interaction between two materials like the connections between atoms in molecules. In this case, the interactions between the atoms of different layers are very strong (chemical bonds). Meanwhile, the melting point of the latter type of hybrid materials is higher than the former one. Note that the interactions between graphene layer and h-BN in this study are the van der Waals ones. Therefore, the repulsive and attractive forces between two layers are one of the reasons affecting on the hardness of graphene, which leads to the lower energy for breaking bonds between carbon atoms in graphene layer in comparison to the free-standing graphene.

In this study, the melting points of graphene layer in hybrid models of graphene/h-BN and graphene/h-BN/graphene models are obtained at around $4550 \mathrm{~K}$ and $5150 \mathrm{~K}$, respectively. Note that the experimentally melting point of free-standing graphene is not presented yet. However, the melting point of graphite which is made out of graphene layers is presented from $4000 \mathrm{~K}$ to 

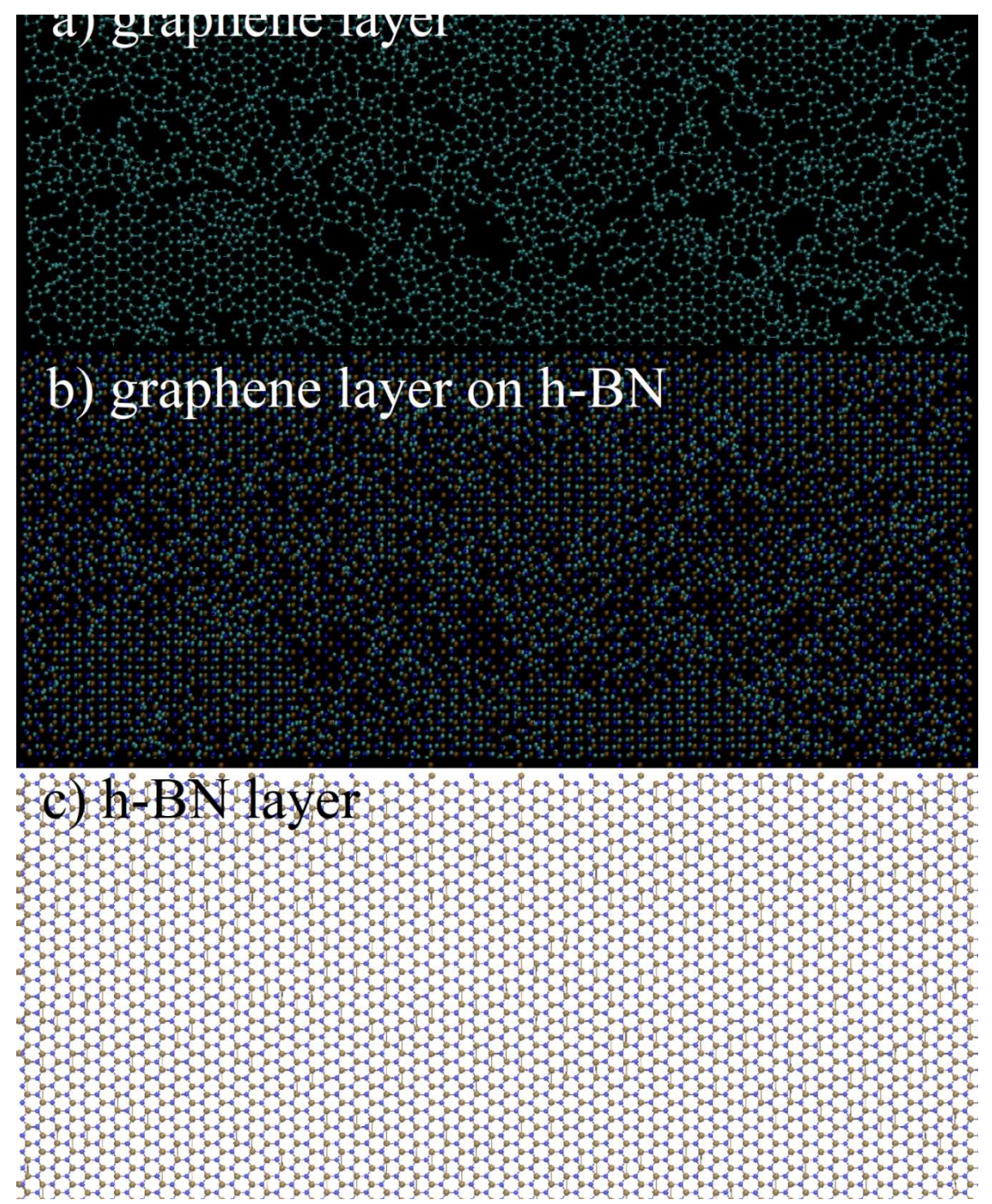

Fig. 2. A side view of 3D visualization of graphene/h-BN model with 5000 atom/layer: a) graphene layer, b) hybrid graphene/h-BN model, c) h-BN layer.

$5000 \mathrm{~K}$ [52]. We included the melting points of free-standing graphene in simulation as well as graphite in experiment here as an additional information to have a general view about graphene.

Radial distribution functions (RDFs) are function of distance between two atoms, which can be used to study the change of graphene structure upon heating. In this study, the RDFs of graphene layer in graphene/h-BN model are presented first to see the evolution of graphene structure (Fig. 3), after that some temperature points are chosen to compare the RDFs of graphene/h-BN model with the ones of graphene/h-BN/graphene model (Fig. 4). 
One can see that the behavior of peaks in the RDF lines decreases and becomes smooth with increasing the temperature (Fig. 3). At temperature near 300K, the RDF exhibits crystalline structure of the model because it contains many peaks (300K line in Fig. 3). When the temperature increases to $2300 \mathrm{~K}$ and $3700 \mathrm{~K}$, although the peaks of RDFs decrease, they are still pointy. At temperature around the melting point $(4600 \mathrm{~K})$, the peaks become smooth that indicates the vibration of atoms in the model $(4600 \mathrm{~K}$ line in Fig. 3). Therefore, it can be considered that the phase transition from solid to liquid states is around this temperature $(4600 \mathrm{~K})$. This temperature point is close to the one that is defined by heat capacity (Fig. 1). The peaks almost disappear at temperature of $5600 \mathrm{~K}$ which indicates that the model is in a liquid state (5600K line in Fig. 3).

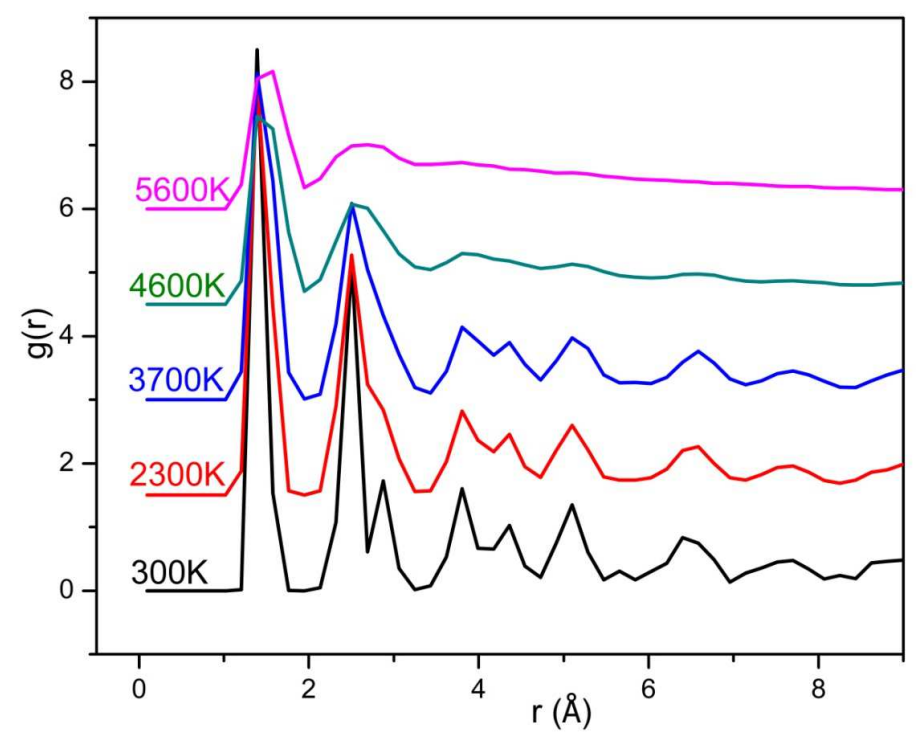

Fig. 3. Temperature dependence of RDF of graphene layer in graphene/h-BN model.

The RDFs of graphene/h-BN and graphene/h-BN/graphene models are presented in Fig. 4. At temperature of $4500 \mathrm{~K}$, the peaks of graphene $/ \mathrm{h}-\mathrm{BN}$ model decrease significant indicating that the model is in a liquid state (the 4500K-solid line in Fig. 4). However, the RDFs of graphene/h$\mathrm{BN} /$ graphene models are still high exhibiting a solid state at this temperature (the $4500 \mathrm{~K}$-dot line in Fig. 4). At temperature of 5500K, the first peak of graphene/h-BN/graphene model decreases sharply and the left peaks are almost smooth indicating that the atoms distribute randomly in the space of the model and the model is in a liquid state (the 5500K-solid line in Fig. 4). One can say that the melting point of graphene/h-BN/graphene model is higher than the one of graphene/h-BN model.

In order to observe the dependence of melting process on the simulation domain size, two graphene/h-BN models with different sizes of each layer (5000 and 10000 atom/layer) are chosen to study (Fig. 5). In term of temperature, the behavior of total energy of models 5000 atoms and 10000 atoms almost coincides: the total energy of both square and circle symbols increases linearly at temperature below $4500 \mathrm{~K}$ before jumping sharply at temperature around $4500 \mathrm{~K}$. After 


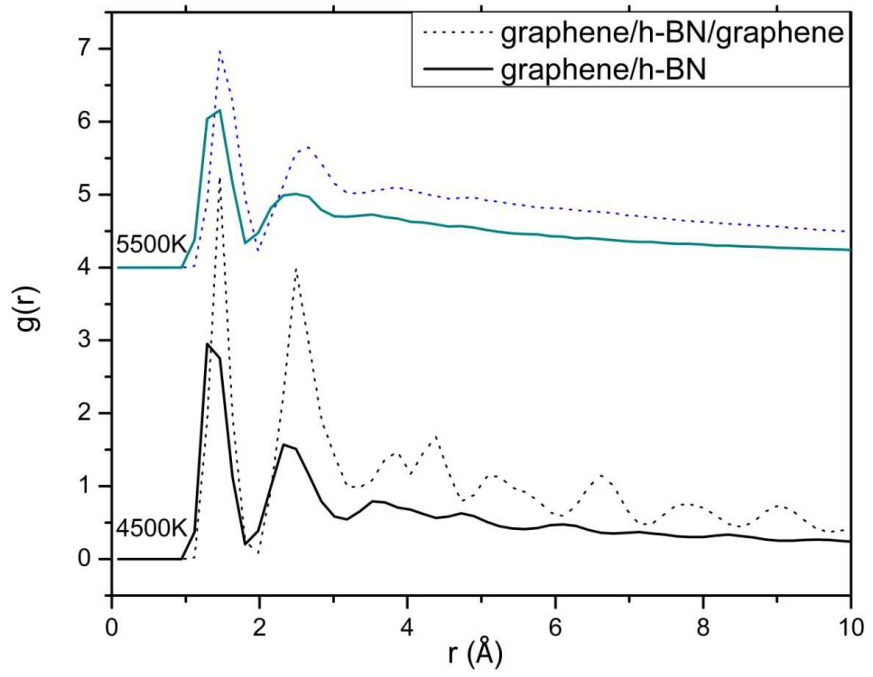

Fig. 4. Temperature dependence of RDFs upon heating: solid lines - graphene/h-BN model, dot lines - graphene/h-BN/graphene model.

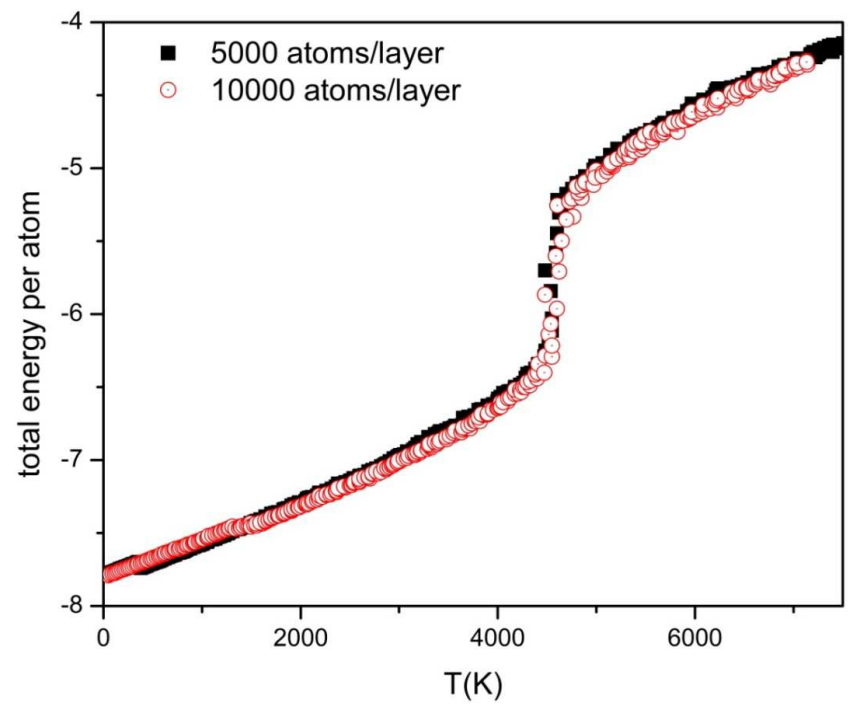

Fig. 5. Temperature dependence of total energy per atom of graphene layer in graphene/h-BN (two layers) model: square symbol - 5000 atom/layer, empty circle symbol - 10000 atom/layer [53].

that the total energy per atom follows the linear law. In term of energy, the statistical noise of the model with 5000 atom/layer (square symbol in Fig. 5) is a bit higher than the one of the model with 10000 atoms per layer (circle dot in Fig. 5). Therefore, in the range of this study, the number of 
atom per layer has tendency to effect on the total energy per atom. In this case, it can be suggested that the difference in size between two models is not large enough to observe the size dependence of the melting points. In addition, the conditions of boundary to create two-dimensional material (i.e. non-periodic boundary condition is applied only along $z$ axis in Cartesian Coordinates) also contributes to this case.

The RDFs of graphene layer in the model with 10000 atoms per layer also present show the difference with the ones of graphene layer in the model with 5000 atoms per layer (solid line - graphene layer with 5000 atoms, dot line - graphene layer with 10000 atoms) (Fig. 6).

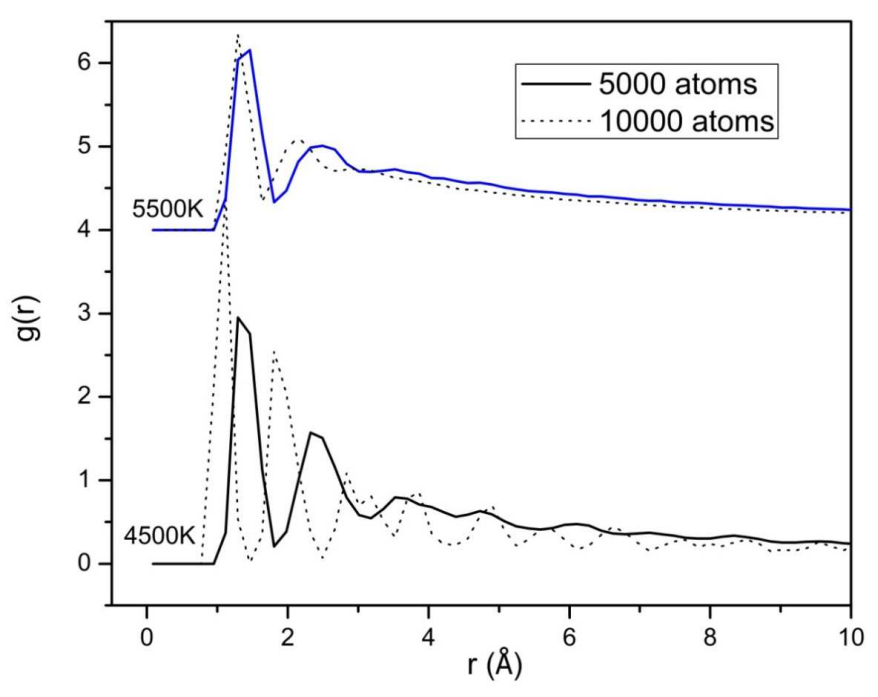

Fig. 6. Temperature dependence of RDFs upon heating: solid lines - graphene/h-BN model with 5000 atoms per layer, dot lines - graphene/h-BN with 10000 atoms per layer [53].

At temperature of $4500 \mathrm{~K}$, the peaks of RDFs of graphene layer with 5000 atoms decrease significantly (solid line at $4500 \mathrm{~K}$ in Fig. 6). The peaks of graphene layer with 10000 atoms are also low from the third peak in comparison with the first and the second ones (dot line at $4500 \mathrm{~K}$ in Fig. 6). In addition, the first and the second peaks tend to move to the shorter distance (dot line at $4500 \mathrm{~K}$ in Fig. 6). It indicates that at $4500 \mathrm{~K}$ the crystal structure of graphene layer with 5000 atoms as well as 10000 atoms per layer is distorted or broken, which leads to the phase transition from solid to liquid states. One can see that at $5000 \mathrm{~K}$, the RDFs of graphene layer in different sizes of models as well as different layers are similar i.e the models are in a liquid state (Figs. $4,6)$. In addition, the number of liquid atoms of graphene layer with 5000 atoms per layer is presented below (Fig. 7). It can be seen from Fig. 7 that at the range of temperature from $4000 \mathrm{~K}$ to $5000 \mathrm{~K}$ there exists simultaneous liquid and solid phases in the model of 5000 atoms per layer. From temperature $5000 \mathrm{~K}$ and higher, the number of liquid atoms increases dramatically, which indicates a liquid state of the model. 


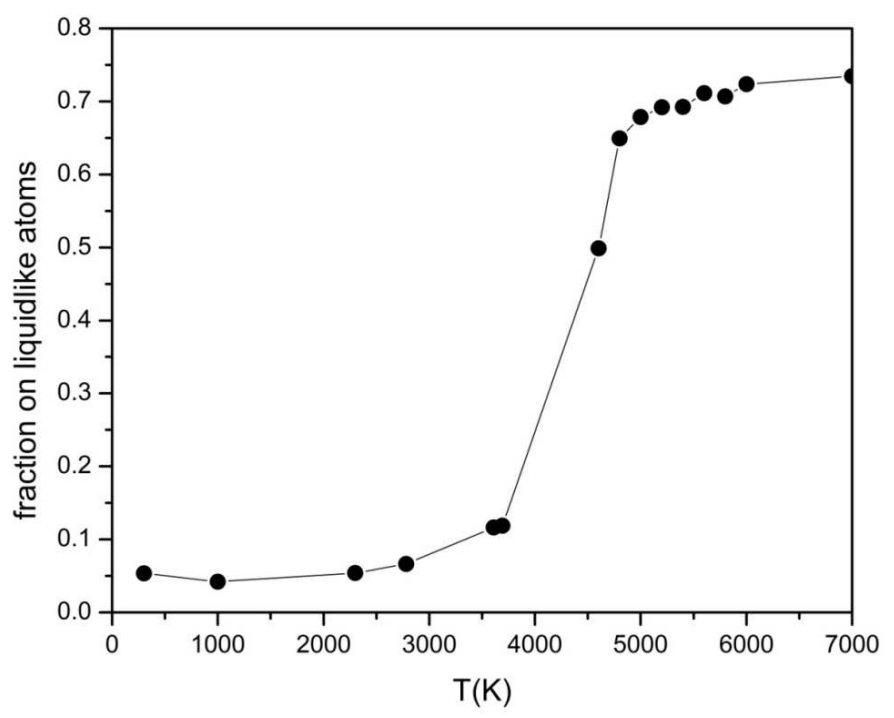

Fig. 7. The liquid-like atoms of graphene layer in hybrid graphene/h-BN model with 5000 atom/layer upon heating.

Note that the similar situation also happens to the liquid atoms of model with 10000 atoms per layer [53]. One can conclude that in the range of this study the melting process does not depend on the size of model.

\section{CONCLUSIONS}

Graphene layer of hybrid models is studied via molecular dynamics simulation. Models with different numbers of layers as well as atoms are studied via Tersoff and Lennard-Jones potentials to have an entire picture about graphene layer upon heating. The melting temperature range of graphene layer in hybrid graphene/h-BN model with 5000 atom/layer is defined around $4550 \mathrm{~K}$. This point of temperature $(4550 \mathrm{~K})$ is close to the one of experiment for graphite. In the range of this study, the melting temperature range depends on the number of layers in the model: the melting temperature range of three layers (graphene/h-BN/graphene) model is around $5150 \mathrm{~K}$ while the one of two layer (graphene/h-BN) model is $4550 \mathrm{~K}$. The phase transition exhibits the first order behavior and satisfies the first step towards Devil's staircase type phase transition.

\section{ACKNOWLEDGEMENTS}

This research is funded by Ho Chi Minh City University of technology - VNU-HCM under grant number T-KHUD-2018-85.

\section{REFERENCES}

[1] D. Jin-Xiang, Z. Xiao-Kang, Y. Qian, W. Xu-Yang, C. Guang-Hua, and H. De-Yan, Chin. Phys. B 18 (2009) 4013. 
[2] C. Li, Y. Bando, C. Zhi, Y. Huang, and D. Golberg, Nanotechnology 20 (2009) 385707.

[3] K. Suenaga, C. Colliex, N. Demoncy, A. Loiseau, H. Pascard, and F. Willaime, Science 278 (1997) 653.

[4] W.Q. Han, W. Mickelson, J. Cumings, and A. Zettl, Appl. Phys. Lett. 81 (2002) 1110.

[5] T. Kawasaki, T. Ichimura, H. Kishimoto, A. A. Akbar, T. Ogawa, and C. Oshima, Surf. Rev. Lett. 9 (2002) 1459.

[6] T. Martins, R. d. Miwa, A.J. Da Silva, and A. Fazzio, Phys. Rev. Let. 98 (2007) 196803.

[7] A. Lherbier, X. Blase, Y.-M. Niquet, F. Triozon, and S. Roche, Phys. Rev. Let. 101 (2008) 036808.

[8] X. Wang, X. Li, L. Zhang, Y. Yoon, P.K. Weber, H. Wang, J. Guo, and H. Dai, Science 324 (2009) 768.

[9] R. Kaner, J. Kouvetakis, C. Warble, M. Sattler, and N. Bartlett, Mater. Res. Bull. 22 (1987) 399.

[10] A.Y. Liu, R.M. Wentzcovitch, and M.L. Cohen, Phys. Rev. B 39 (1989) 1760.

[11] Y. Miyamoto, A. Rubio, M.L. Cohen, and S.G. Louie, Phys. Rev. B 50 (1994) 4976.

[12] Z. Weng-Sieh, K. Cherrey, N.G. Chopra, X. Blase, Y. Miyamoto, A. Rubio, M.L. Cohen, S.G. Louie, A. Zettl, and R. Gronsky, Phys. Rev. B 51 (1995) 11229.

[13] E. Hernandez, C. Goze, P. Bernier, and A. Rubio, Phys. Rev. Lett. 80 (1998) 4502.

[14] D. Golberg, Y. Bando, L. Bourgeois, K. Kurashima, and T. Sato, Carbon 38 (2000) 2017.

[15] D. Golberg, Y. Bando, P. Dorozhkin, and Z.-C. Dong, Mater. Res. Bull. 29 (2004) 38.

[16] M. Bokdam, P.A. Khomyakov, G. Brocks, Z. Zhong, and P.J. Kelly, Nano Lett. 11 (2011) 4631.

[17] C. R. Dean, A. F. Young, I. Meric, C. Lee, L. Wang, S. Sorgenfrei, K. Watanabe, T. Taniguchi, P. Kim, and K.L. Shepard, Nat. Nanotechnol. 5 (2010) 722.

[18] M. Son, H. Lim, M. Hong, and H.C. Choi, Nanoscale 3 (2011) 3089.

[19] S. Tang, G. Ding, X. Xie, J. Chen, C. Wang, X. Ding, F. Huang, W. Lu, and M. Jiang, Carbon 50 (2012) 329.

[20] S. Tang, H. Wang, Y. Zhang, A. Li, H. Xie, X. Liu, L. Liu, T. Li, F. Huang, and X. Xie, Sci. Rep. 3 (2013) 2666.

[21] W. Yang, G. Chen, Z. Shi, C. C. Liu, L. Zhang, G. Xie, M. Cheng, D. Wang, R. Yang, and D. Shi, Nat. Mater. 12 (2013) 792.

[22] S. Tang, H. Wang, H.S. Wang, Q. Sun, X. Zhang, C. Cong, H. Xie, X. Liu, X. Zhou, and F. Huang, Nat. Commun. 6 (2015) 6499.

[23] K. K. Kim, A. Hsu, X. Jia, S. M. Kim, Y. Shi, M. Hofmann, D. Nezich, J. F. Rodriguez-Nieva, M. Dresselhaus, and T. Palacios, Nano Lett. 12 (2011) 161.

[24] L. Gao, W. Ren, H. Xu, L. Jin, Z. Wang, T. Ma, L.P. Ma, Z. Zhang, Q. Fu, and L.M. Peng, Nat. Commun. 3 (2012) 699.

[25] G. Kim, A. R. Jang, H. Y. Jeong, Z. Lee, D. J. Kang, and H. S. Shin, Nano Lett. 13 (2013) 1834.

[26] H. Arjmandi-Tash, D. Kalita, Z. Han, R. Othmen, G. Nayak, C. Berne, J. Landers, K. Watanabe, T. Taniguchi, and L. Marty, J.Phys. Materials. 1 (2018) 015003.

[27] M. Kawaguchi, T. Kawashima, and T. Nakajima, Chem. Mater. 8 (1996) 1197.

[28] L. Ci, L. Song, C. Jin, D. Jariwala, D. Wu, Y. Li, A. Srivastava, Z. Wang, K. Storr, and L. Balicas, Nat. Mater. 9 (2010) 430.

[29] F. H. Stillinger and T.A. Weber, Phys. Rev. B 31 (1985) 5262.

[30] M. Z. Bazant, E. Kaxiras, and J. F. Justo, Phys. Rev. B 56 (1997) 8542.

[31] J. F. Justo, M. Z. Bazant, E. Kaxiras, V. V. Bulatov, and S. Yip, Phys. Rev. B 58 (1998) 2539.

[32] N. Marks, Phys. Rev. B 63 (2000) 035401.

[33] M. Finnis and J. Sinclair, Philos. Mag. A 50 (1984) 45.

[34] M. Baskes, Phys. Rev. Let. 59 (1987) 2666.

[35] D. Pettifor, Phys. Rev. Let. 63 (1989) 2480.

[36] D. Pettifor and I. Oleinik, Phys. Rev. B 59 (1999) 8487.

[37] D. W. Brenner, O. A. Shenderova, J. A. Harrison, S. J. Stuart, B. Ni, and S. B. Sinnott, J. Phys.: Condens. Mat. 14 (2002) 783.

[38] A. C. Van Duin, S. Dasgupta, F. Lorant, and W.A. Goddard, J. Phys. Chem. A 105 (2001) 9396.

[39] A. C. Van Duin, A. Strachan, S. Stewman, Q. Zhang, X. Xu, and W.A. Goddard, J. Phys. Chem. A 107 (2003) 3803.

[40] K. Chenoweth, A.C. Van Duin, and W.A. Goddard, J. Phys. Chem. A 112 (2008) 1040.

[41] J. Tersoff, Phys. Rev. B 37 (1988) 6991.

[42] J. Tersoff, Phys. Rev. B 39 (1989) 5566.

[43] J. Nord, K. Albe, P. Erhart, and K. Nordlund, J. Phys.: Condens. Mat. 15 (2003) 5649. 
[44] D. W. Brenner, Phys. Rev. B 42 (1990) 9458.

[45] N. Yu and A. A. Polycarpou, J. Colloid Interface Sci. 278 (2004) 428.

[46] J.W. Kang and H.J. Hwang, J. Phys.: Condens. Mat. 16 (2004) 3901.

[47] S. Plimpton, J. Comput. Phys. 117 (1995) 1.

[48] V. V. Hoang, L. T. Cam Tuyen, and T. Q. Dong, Philos. Mag. 96 (2016) 1993.

[49] S. Gleiman, C.-K. Chen, A. Datye, and J. Phillips, J. Mater. Sci. 37 (2002) 3429.

[50] W. Humphrey, A. Dalke, and K. Schulten, J. Mol. Graphics 14 (1996) 33.

[51] P. Bak, Rep. Prog. Phys. 45 (1982) 587.

[52] A. Savvatimskiy, Carbon 43 (2005) 1115.

[53] Hang T. T. Nguyen, Carbon Lett. 29 (2019) 521. 